\title{
"My friend who bought it for me, she has had an abortion before." The influence of Ghanaian women's social networks in determining the pathway to induced abortion
}

\author{
Sarah D Rominski, ${ }^{1}$ Jody R Lori, ${ }^{2}$ Emmanuel SK Morhe ${ }^{3}$
}

\begin{abstract}
'Department of Obstetrics and Gynecology, University of Michigan, Ann Arbor, MI, USA ${ }^{2}$ Department of Health Behavior and Biological Sciences, University of Michigan School of Nursing, Ann Arbor, MI, USA

${ }^{3}$ Kwame Nkrumah University of Science and Technology, Kumasi, Ghana
\end{abstract}

\section{Correspondence to}

Dr Sarah D Rominski, Univ Michigan, 1111 E. Catherine St. Ann Arbor 48109, Michigan, USA; sarahrom@umich.edu

Received 18 March 2016 Revised 20 January 2017 Accepted 6 March 2017 Published Online First 22 March 2017

CrossMark

To cite: Rominski SD, Lori JR, Morhe ESK. J Fam Plann Reprod Health Care 2017:43:216-221.

\begin{abstract}
Background Even given the liberal abortion law in Ghana, abortion complications are a large contributor to maternal morbidity and mortality. This study sought to understand why young women seeking an abortion in a legally enabling environment chose to do this outside the formal healthcare system.

Methods Women being treated for complications arising from a self-induced abortion as well as for elective abortions at three hospitals in Ghana

\section{Key message points}

- Women in Ghana consult members of their social networks when faced with an unwanted pregnancy.

- Due to multiple factors, including the wide availability of misoprostol, the poor quality of care women receive in facilities, and wrongly believing abortion is illegal, self-inducing abortions has been normalised.
\end{abstract} were interviewed. Community-based focus groups were held with women as well as men, separately. Interviews and focus group discussions were conducted until saturation was reached.

Results A total of 18 women seeking care for complications from a self-induced abortion and 11 seeking care for an elective abortion interviewed. The women ranged in age from 13 to 35 years. There were eight focus groups; two with men and six with women. The reasons women self-induce are: (1) abortion is illegal; (2) attitudes of the healthcare workers; (3) keeping the pregnancy a secret; and (4) social network influence. The meta-theme of 'normalisation of self-inducing' an abortion was identified.

Discussion When women are faced with an unplanned and unwanted pregnancy, they consult individuals in their social network whom they know have dealt with a similar situation. Misoprostol is widely available in Ghanaian cities and is successful at inducing an abortion for many women. In this way, self-inducing abortions using medication procured from pharmacists and chemical sellers has become normalised for women in Kumasi, Ghana.

\section{INTRODUCTION}

Unsafe abortion is a major cause of maternal mortality. Complications from unsafe abortion cause approximately $10 \%$ of maternal deaths in sub-Saharan Africa, ${ }^{1}$ although these numbers are likely underreported in areas where abortion is illegal and/or highly stigmatised. ${ }^{2}$ An unsafe abortion is defined by the World Health Organization (WHO) as a procedure which is provided by a person who has not been trained or in a location which does not meet minimal medical standards, or both. ${ }^{3}$ Deaths resulting from unsafe abortion exist almost exclusively in low-income countries where there are often highly restrictive laws governing abortion provision and where there are scant providers trained and willing to provide safe procedures. ${ }^{4}$ Abortion-related mortality remains high in many sub-Saharan African countries where abortions are legally restricted, and while the overall number of abortions is reducing worldwide, the number of unsafe abortions is increasing. ${ }^{5}$

In countries where abortion laws are restrictive, women faced with an unwanted pregnancy have little choice but to induce clandestinely and often 
unsafely. Ghana, however, has an abortion law that the Guttmacher Institute calls 'liberal'. ${ }^{6}$ Beyond the legal framework, the Ghana Health Service has made access to safe abortion care part of its policy. Safe abortion, performed by a qualified healthcare provider, has been part of the Reproductive Health Strategy since $2003 .{ }^{78}$ Currently in Ghana, abortion is a criminal offence regulated by Act 29, Section 58 of the Criminal code of 1960, amended by PNDCL 102 of 1985 . $^{7}$ However, Section 2 of this law states abortion may be performed by a registered medical practitioner when: the pregnancy is the result of rape or incest, to protect the mental or physical health of the mother, or when there is a malformation of the fetus. The government of Ghana has taken steps to mitigate the negative effects of unsafe abortion by developing a comprehensive reproductive health strategy that specifically addresses maternal morbidity and mortality associated with unsafe abortion. ${ }^{9}$ Despite these steps, however, abortion complications are a large contributor to maternal morbidity and mortality. ${ }^{10} 11$ Complications from unsafe abortion are the leading cause of maternal mortality for young women (aged 19 years and below) and the second leading cause of maternal mortality for all women of reproductive age. ${ }^{12}$

Given the liberal legal and policy framework surrounding access to safe abortion in Ghana, this study sought to understand the perspective of women who decide to terminate their pregnancy in a manner the WHO and other international organisations classify as unsafe.

\section{METHODS}

\section{Research design and participants}

Using a qualitative, grounded theory design, interviews and focus groups were conducted. Women meeting the inclusion criteria (receiving treatment for complications arising from a self-induced abortion or coming for an elective induced abortion) at three hospitals in the Ashanti region of Ghana were offered participation in the study by the nurse-midwife on duty. If the woman was interested, the research assistant came to the hospital to interview the participant. All women were informed of the research objectives and were assured of confidentiality and anonymity. No identifying information was collected. All of the participants were informed of the voluntary nature of the research and were interviewed only after their treatment was completed and before they were discharged.

Additionally, focus group discussions (FGDs) with between six and 10 participants were held in the community to further contextualise how members of the community conceptualise and access abortion services in the case of unwanted pregnancies. Participants were approached by a research assistant and asked if they would like to participate and voluntarily recruited.
The interview and focus groups guides were openended and included probes for additional issues that could emerge as concerns or issues arouse. Some of the key categories explored included: women's and community members' perception of the legal status of abortion in Ghana; comfort-seeking care in a hospital or health centre for abortion care; use of contraception; and the existence of community-based abortion providers. The interview and focus-group guides were pilot-tested in advance among a smaller sample from the target population and revised to ensure flow and clarity.

Ethical approval was received by the University of Michigan Institutional Review Board (HUM00087225) and the Komfo Anokye Teaching Hospital/Kwame Nkrumah University of Science and Technology Committee on Human Research Publication and Ethics. All participants gave verbal informed consent, and for those who were under the age of 18 years, parental consent was given. All participants were given a written participant information sheet and informed of the confidentiality of the research.

\section{Data collection procedure}

The individual interviews took place in a private room near the ward where the women were being treated. Only the participant and the research assistant were in the room. The focus groups were held with women and men separately, in market areas where participants were generally engaged in their daily work, allowing people from different communities and other social background to be recruited. Potential participants were approached by members of the study team and the purpose of the study was described. Those who were interested in participating were taken through a comprehensive consent procedure. The FGDs were held in various locations away from others at times when the markets were not particularly busy, both for privacy as well as for quality recording. The interviews and focus groups were conducted and recorded in the local language, Twi, and transcribed in English by a research assistant fluent in both languages. Transcription was conducted on an ongoing schedule, with the transcripts being reviewed by the study team at regular intervals and data capture continued until saturation was reached.

\section{Data analysis}

Qualitative data were reviewed in Microsoft Word (Microsoft Corp., Redmond, WA) by the study team. Descriptive statistical and qualitative analyses were performed using Microsoft Excel and SPSS Version 22.0 (IBM Corp., Armonk, NY). The qualitative data were analysed using the constant comparative approach to identify key themes. ${ }^{13}$ The researchers undertook line-by-line coding of the transcripts, after initial high-level reading of all the transcripts. From the initial coding, a large number of codes emerged. From this large number of initial codes, reappearing 
and similar codes were identified and consolidated. When coding discrepancies emerged, they were discussed until consensus was reached. These codes were then raised to the level of categories and the categories were grouped together into themes. Quotes that were particularly illustrative of these themes were selected. Finally, one meta-theme emerged from the data.

\section{RESULTS}

There were 18 women seeking care for complications from a self-induced abortion (PAC) and 11 seeking care for an elective abortion (CAC) interviewed. The women ranged in age from 13 to 35 years, with a mean age of 25.7 years. There were eight focus groups; two with men and six with women. The focus groups had between six and 10 participants each.

The reasons for self-inducing among some women are grouped into four themes. These themes are: (1) legal status of abortion; (2) attitudes of the healthcare workers; (3) keeping the pregnancy a secret; and (4) social network influence. Finally, a meta-theme of the 'normalisation of self-inducing' emerged from these data.

\section{Legal status of abortion}

The legal status of abortion was mentioned by all groups of participants as a reason for why women self-induce rather than come to facilities for abortion services. Prosecution of women, or their providers, due to inducing an abortion is rare,${ }^{14}$ but women are afraid of this potentiality. As one woman said, "If they had seen me, then I would have been arrested but now that no one has seen me, I think I am safe". [32-year-old PAC client, district hospital]

When asked the reasons a woman in Ghana can have a legal abortion, a woman being treated for post-abortion complications said, "Well, I know abortions are against the law in Ghana". [PAC client, teaching hospital].

Although the majority of participants in this study, and all who were being treated for complications after self-inducing their abortion, wrongly believed abortion is illegal in Ghana, others had a good understanding of the law. As one client seeking care for an induced abortion said, "I don't think so [that abortion is illegal] because I know at the hospitals one can obtain the service”. [22-year-old CAC client, district hospital]. Another client was even more sure: "Abortion is legal ... most women don't know. I had information from a druggist who is a nurse". [27-year-old CAC client, district hospital] Another said, "I have heard about the legality of abortion ... through health talks at the post natal and child welfare clinic”. [35-year-old, CAC client]

\section{Attitudes of the health workers}

Many participants noted the 'attitude' of the health workers and how women are treated by staff impact women's willingness to patronise services, as evidenced by this quote: "Even for me it [rude treatment by healthcare providers] is the reason why I stayed away from the hospital". [28-year-old, PAC client, district hospital]

One focus group participant said: "There was a case recently where the girl [who needed an abortion] was afraid of being mistreated so she resorted to taking drugs and unfortunately she died". [female FGD]

In response to a question about what a woman with an unwanted pregnancy should do, a male participant of a focus group said, "I think that she should go to the hospital". However, he further stated, "they are often scared, because if they go there, they are usually mistreated by the nurses". [male FGD]

While poor treatment by staff is not isolated to care being provided for abortion services, it is perhaps especially pronounced for this stigmatised service.

\section{Keeping the pregnancy a secret}

Many women self-induce in order to keep their pregnancy a secret, as evidenced by this quote: "I didn't want my mother to know that is why I tried to terminate myself". [23-year-old, PAC client, district hospital]

While some women know they can get an abortion from a clinic, because they want to do it privately without many people knowing, they are more likely to induce at home, and seek care if there are complications. The following interaction during one of the community focus groups demonstrates this:

Interviewer: "When the girls or ladies want to abort their pregnancies, what do they do?"

Respondent: "In my community, there are some individuals who are believed to have special skills when it comes to aborting pregnancies, so they go to them and then some herbal mixtures and concoctions are prepared for them."

Interviewer: "Do they ever remember that there is a clinic there that they can seek help from?"

Respondent: "Yes they know that there is a clinic available, but because they don't want any soul to know of their state, they sneak to these people for the procedure." [female FGD]

\section{Social network influence}

Women in the study reported learning how to terminate their pregnancies while avoiding the formal healthcare system from their friends or others in their social network. Women discuss their unwanted pregnancies with others they know have also had unwanted pregnancies, seeking advice and guidance.

"The girls involved in abortion get their information from friends, neighbours and all manner of people. All that a girl needs is to tell her problem to someone and the next time, she is being shown where she can get help from. They tell them to buy all sorts of things." [female FGD] 
"They go to people who have done abortion before and then they inquire about how they manage to get rid of their pregnancies and if they have any help that they can give to them so that they too can get rid of their pregnancy." [27 year-old PAC client, district hospital]

Many women use a combination of advice from friends and covert purchase from a chemical seller. Friends are often enlisted to do the actual purchasing and perhaps even the dispensing of advice on how to take the drug. A young client being treated for post-abortion complications tells of how she obtained the drug she used:

Interviewer: "What drug did you use?"

Respondent : "I don't know the name."

Interviewer: "Where did you buy it from?"

Respondent: "It was my friend who bought it for me... My friend who bought it for me, she has had an abortion before." [20-year-old PAC client, district hospital]

Women take drugs without knowing what they are, only that their friend or boyfriend has told them it will terminate the pregnancy. Those participants who had come to the facilities for an induced abortion also reported learning about where to go from friends or others in their social networks. For example:

"I discussed with my sister and husband. My sister said a certain lady also once had an unwanted pregnancy and told her she went to [District Hospital] for the termination so she would take me to that person. My sister's friend as I have already told you, showed me the place because she also had her problem solved here." [32-year-old CAC client, district hospital]

\section{Normalisation of self-inducing}

The meta-theme which emerged from these data is the idea that, due to many of the themes elucidated on above, it has become the normal course of action for many women with an unwanted pregnancy to self-induce an abortion. As one participant of a community-based focus group said: "[Women] are of the view that if they take the medication at home, it wouldn't bring any problem to them." [female FGD]

If complications do arise, women seek care from a health facility. As one participant of a focus group said in response to a question of whether the local health facility provides abortion services: "No. But usually when ladies suffer complications, they are rushed there and given treatment." Many women prefer to induce their abortion with the help of friends at home, and only seek services at the health facilities if there is a problem.

\section{DISCUSSION}

In order for women to have access to safe abortion services, there needs to be an enabling legal environment and well-trained providers who are willing to provide the service close to where women live. ${ }^{15}$ The government of Ghana has taken steps to bring these services to its population. The law governing abortion was liberalised in 1985, and in 2006 the Ghana Health Service published the Standards and Protocols document to "facilitate the provision of a package of services called Comprehensive Abortion Care that will reduce unwanted pregnancy and abortion-related maternal morbidity and mortality". ${ }^{16}$ However, there are still many women who choose to self-induce rather than seek services in a facility. Some women in this study were sure that abortion was illegal, a few thought it was legal, and many did not know. This is a difference between those seeking care for CAC and those seeking care for PAC. Understanding the law influenced choices that respondents made. Those who thought abortion was legal sought elective abortion while those who thought abortion was illegal had unsafe abortion and sought PAC for treatment of complications. The current law may not be straightforward to some and abortion is still technically illegal.

A negative attitude and rude or hostile behaviour on the part of healthcare providers was mentioned by participants as reasons women choose to self-induce their abortions. As has been reported elsewhere, both in Ghana and in other African settings, ${ }^{17}$ women are judged negatively by providers if they seek an abortion. Ensuring providers do not push their own judgments onto a client is an integral component of values clarification training in which providers go through exercises to understand their own biases and learn strategies to provide non-judgmental care. ${ }^{18}$ While values clarification will not immediately change all provider's deeply held negative attitudes towards abortion services and the women who seek these services, this is an important initial step to begin to improve the quality of care women who seek abortion services receive. Further research is needed on both measuring provider willingness to provide abortion services and other forms of training that are effective at increasing provider willingness.

All of the women in this study who self-induced used medication to do so. Medication abortion using misoprostol has been shown to be safe and up to $94 \%$ effective for early pregnancy termination. ${ }^{19}$ In countries where abortion is either illegal or highly restricted, medication abortion has provided women a safe and discreet way to terminate unwanted pregnancy. ${ }^{20}$ It is becoming clear that medication abortion is reducing women's dependence on medical systems, providing them with greater autonomy and control over their most important reproductive decisions. ${ }^{21}$ Misoprostol is both inexpensive and stable at room temperature, two features which make it ideal for low-income countries where refrigeration may not be possible. ${ }^{22} 23$ With the introduction of misoprostol in many low-income settings, there is a potential to dramatically reduce abortion-related deaths. ${ }^{24}$

Misoprostol has been identified as a potentially safer way for women to induce abortions in localities where abortion is highly restricted, and WHO has endorsed 
the home use of misoprostol after clinic-based provision of mifepristone stating this can improve "privacy, convenience and acceptability of services, without compromising on safety" for early pregnancies ${ }^{25}$ (p. 28). However, there are also indications that when women are reluctant to seek care for induced abortion due to stigma or poor quality of care from health workers, misoprostol might be a safer alternative than crude surgical methods or local preparations ${ }^{26}$. It is possible that women in Ghana, while inducing outside a health facility, are not experiencing 'unsafe' abortions as they had been conceptualised previously. With the introduction of misoprostol, the dichotomy of safe versus unsafe is potentially being challenged. The women being treated for complications resulting from a self-induced abortion were not using crude surgical methods, but were rather self-inducing using the same drug they would be provided had they sought their abortion in a facility.

\section{LIMITATIONS}

This study has some important limitations. First, the research team was made up of educated researchers who are socially distant from most of the participants in the study. This distance may have made participants less likely to discuss some important aspects of careseeking for a service which is stigmatised. Also, the study was based solely on self-report. There were no objective measures of the use of unsafe abortion procedures. Although women did not have an incentive to tell anything but the truth, it was not possible to verify where they obtained the drugs they used, for example. Further only women who eventually were treated in a facility were included. This may miss a portion of the population who would not choose to seek care in a facility for complications, those who were unable to due to the serious nature of their complications, or those who successfully self-induced their abortion without complications using misoprostol. Those women who were convinced to carry an unwanted pregnancy to term were also not included in these data. By including FGDs, we attempted to triangulate findings; however, recall may be less accurate in a community-based setting than for women have recently dealt with an unwanted pregnancy. Finally, the relatively small sample size reduces the ability to generalise these findings. Qualitative methods were chosen to allow the researchers to delve more deeply into the context of self-induced abortion in this locality, but a larger study would offer different evidence.

\section{CONCLUSIONS}

Although Ghana has a relatively liberal law governing abortion, many women are choosing to self-induce rather than seek care in a registered health facility. There is no one reason for this and therefore there is no one solution. While the Ghana Health Service has made it clear that they are interpreting the existing law in a liberal manner, many Ghanaians are under the impression that abortions are not available legally. Poor quality of care has also plagued the public health system, and women are thus reluctant to seek services for which they may receive sub-standard care. When women are faced with an unplanned and unwanted pregnancy, they consult individuals in their social network whom they know have dealt with a similar situation. In this way, self-induced abortions using medication procured from pharmacists and chemical sellers, has become normalised for women in Kumasi, Ghana.

Correction notice This paper has been amended since it was published Online First. Owing to a scripting error, some of the publisher names in the references were replaced with 'BMJ Publishing Group'. This only affected the full text version, not the PDF. We have since corrected these errors and the correct publishers have been inserted into the references.

Competing interests None declared.

Provenance and peer review Not commissioned; externally peer reviewed.

(C) Faculty of Sexual and Reproductive Healthcare of the Royal College of Obstetricians and Gynaecologists (unless otherwise stated in the text of the article) 2017. All rights reserved. No commercial use is permitted unless otherwise expressly granted.

\section{REFERENCES}

1 Say L, Chou D, Gemmill A, et al. Global causes of maternal death: a WHO systematic analysis. Lancet Glob Health 2014;2:e323-e333.

2 Nieburg P. Improving Maternal Mortality and Other Aspects of Women's Health. The United States' Global Role. Center for Strategic and International Studies. 2012. http://reliefweb. int/sites/reliefweb.int/files/resources/121003_Nieburg_ MaternalMortality_Web.pdf (accessed 1 June 2016).

3 World Health Organization (WHO). Maternal Health and Safe Motherhood Programme. The Prevention and Management of Unsafe Abortion: Report of a Technical Working Group (WHO/MSM/92.5). Geneva, Switzerland: WHO. 1993 whqlibdoc.who.int/hq/1992/WHO_MSM_92.5.pdf (accessed 1 June 2016).

4 Grimes DA, Benson J, Singh S, et al. Unsafe abortion: the preventable pandemic. Lancet 2006;368:1908-1919.

5 Sedgh G, Singh S, Shah IH, et al. Induced abortion: incidence and trends worldwide from 1995 to 2008. Lancet 2012;379:625-632.

6 Sundaram A, Juarez F, Bankole A, et al. Factors associated with abortion-seeking and obtaining a safe abortion in Ghana. Stud Fam Plann 2012;43:273-286.

7 Morhee R, Morhee E. Overview of the law and availability of abortion services in Ghana. Ghana Med J 2006;40:80-86.

8 Sedge G. Abortion in Ghana. Brief. New York, NY: Guttmacher Institute, 2010. No. 2.

9 Taylor J, Diop A, Blum J, et al. Oral misoprostol as an alternative to surgical management for incomplete abortion in Ghana. Int J Gynaecol Obstet 2011;112:40-44.

10 Asamoah BO, Moussa KM, Stafström M, et al. Distribution of causes of maternal mortality among different sociodemographic groups in Ghana; a descriptive study. BMC Public Health 2011;11:159. 
11 Billings DL, Ankrah V, Baird TL, et al. Midwives and comprehensive postabortion care in Ghana. In: Huntington D, Piet-Pelon NJ, (eds). Postabortion Care: Lessons from Operations Research. New York, NY: Population Council, 1999.

12 Asamoah BO, Moussa KM, Stafström M, et al. Distribution of causes of maternal mortality among different socio-demographic groups in Ghana; a descriptive study. BMC Public Health 2011;11:159.

13 Glaser BG. Basics of Grounded Theory Analysis. Mill Valley, CA: Sociology Press, 1992.

14 Kumi-Kyereme A, Gbagbo FY, Amo-Adjei J. Role-players in abortion decision-making in the Accra Metropolis, Ghana. Reprod Health 2014;11:70.

15 Osman S, Thompson A. Unsafe Abortion in South Africa: A Preventable Pandemic. 2012. http://www.ngopulse.org/blogs/ unsafe-abortion-south-africa-preventable-pandemic (accessed 1 June 2016).

16 Ghana Health Service. Prevention and Management of Unsafe Abortion: Comprehensive Abortion Care Services. Standards and Protocols. June 2006.

17 Schwandt HM, Creanga AA, Danso KA, et al. A comparison of women with induced abortion, spontaneous abortion and ectopic pregnancy in Ghana. Contraception 2011;84:87-93.

18 Mitchell EM, Trueman K, Gabriel M, et al. Building alliances from ambivalence: evaluation of abortion values clarification workshops with stakeholders in South Africa. Afr J Reprod Health 2005;9:89-99.
19 Shochet T, Diop A, Gaye A, et al. Sublingual misoprostol versus standard surgical care for treatment of incomplete abortion in five sub-Saharan African countries. BMC Pregnancy Childbirth 2012;12:127.

20 Hyman A, Blanchard K, Coeytaux F, et al. Misoprostol in women's hands: a harm reduction strategy for unsafe abortion. Contraception 2013;87:128-130.

21 Winikoff B, Sheldon W. Use of medicines changing the face of abortion. Int Perspect Sex Reprod Health 2012;38:164-166.

22 Billings DL. Misoprostol alone for early medical abortion in a Latin American clinic setting. Reprod Health Matters 2004;12(24 Suppl.):57-64.

23 Sayette H, Redwine D, Sivin I, et al. Buccal use of misoprostol alone for early abortion: the experience in four Latin American countries. Contraception 2011;84:304.

24 Harper CC, Blanchard K, Grossman D, et al. Reducing maternal mortality due to elective abortion: potential impact of misoprostol in low-resource settings. Int J Gynaecol Obstet 2007;98:66-69.

25 World Health Organization. Clinical Practice Handbook for Safe Abortion. Mill Valley, CA: Sociology Press, 2014.

26 Grimes DA. Reducing the complications of unsafe abortion: the role of medical technology. In: Shah IH, Warriner IK, eds. Preventing Unsafe Abortion and its Consequences: Priorities for Research and Action. New York, NY: Guttmacher Institute, 2006. 